\title{
Multicentre observational screening survey for the detection of CTEPH following pulmonary embolism
}

\author{
Nicolas Coquoz ${ }^{1,2}$, Daniel Weilenmann ${ }^{3}$, Daiana Stolz ${ }^{4}$, Vladimir Popov ${ }^{5}$, \\ Andrea Azzola ${ }^{6}$, Jean-Marc Fellrath ${ }^{7}$, Hans Stricker ${ }^{8}$, Alberto Pagnamenta ${ }^{9}$, \\ Sebastian Ott ${ }^{10}$, Silvia Ulrich ${ }^{11}$, Sandor Györik ${ }^{12}$, Jérôme Pasquier ${ }^{13}$ and \\ John-David Aubert (10) $1,2,14$
}

Affiliations: ${ }^{1}$ Service de Pneumologie, Centre Hospitalier Universitaire Vaudois (CHUV), Lausanne, Switzerland. ${ }^{2}$ Faculté de Biologie et de Médecine, Université de Lausanne, Lausanne, Switzerland. ${ }^{3}$ Klinik für Kardiologie, Kantonsspital St Gallen, St Gallen, Switzerland. ${ }^{4}$ Clinic of Respiratory Medicine and Pulmonary Cell Research, University Hospital Basel, Basel, Switzerland. ${ }^{5}$ Innere Medizin, Pneumologie und Schlafmedizin, Lungenzentrum Hirslanden, Zürich, Switzerland. ${ }^{6}$ Pneumologia e Medicina Intensiva, Ospedale Civico, Lugano, Switzerland. ${ }^{7}$ Service de Pneumologie, Hôpital de Pourtalès, Neuchâtel, Switzerland. ${ }^{8}$ Servizio di Angiologia, Ospedale La Carità, Locarno, Switzerland. ${ }^{9}$ Reparto di Medecina Intensiva, Ospedale Regionale di Mendrisio, Mendrisio, Switzerland. ${ }^{10} \mathrm{Klinik}$ für Pneumologie, Universitätsspital (Inselspital) und Universität Bern, Bern, Switzerland. ${ }^{11}$ Klinik für Pneumologie, Universitätsspital Zürich, Zürich, Switzerland. ${ }^{12}$ Servizio di Pneumologia, Ospedale Bellinzona e Valli, Bellinzona, Switzerland. ${ }^{13}$ Institut de Médecine Sociale et Préventive, Université de Lausanne, Lausanne, Switzerland. ${ }^{14}$ Centre de Transplantation d'Organes, Centre Hospitalier Universitaire Vaudois (CHUV), Lausanne, Switzerland.

Correspondence: John-David Aubert, Service de Pneumologie et Centre de Transplantation d'Organes, Centre Hospitalier Universitaire Vaudois (CHUV), Rue du Bugnon 46, 1011 Lausanne, Switzerland. E-mail: JohnDavid.Aubertachuv.ch

@ERSpublications

Chronic thromboembolic pulmonary hypertension is an uncommon disease; active screening of CTEPH in patients after acute pulmonary embolism should be considered http://ow.ly/mmFI30j3Lca

Cite this article as: Coquoz N, Weilenmann D, Stolz D, et al. Multicentre observational screening survey for the detection of CTEPH following pulmonary embolism. Eur Respir J 2018; 51: 1702505 [https://doi. org/10.1183/13993003.02505-2017].

ABSTRACT Chronic thromboembolic pulmonary hypertension (CTEPH) is a severe complication of pulmonary embolism. Its incidence following pulmonary embolism is debated. Active screening for CTEPH in patients with acute pulmonary embolism is yet to be recommended.

This prospective, multicentre, observational study (Multicentre Observational Screening Survey for the Detection of Chronic Thromboembolic Pulmonary Hypertension (CTEPH) Following Pulmonary Embolism (INPUT on PE); ISRCTN61417303) included patients with acute pulmonary embolism from 11 centres in Switzerland from March 2009 to November 2016. Screening for possible CTEPH was performed at 6,12 and 24 months using a stepwise algorithm that included a dyspnoea phone-based survey, transthoracic echocardiography, right heart catheterisation and radiological confirmation of CTEPH.

Out of 1699 patients with pulmonary embolism, 508 patients were assessed for CTEPH screening over 2 years. CTEPH incidence following pulmonary embolism was 3.7 per 1000 patient-years, with a 2 -year cumulative incidence of $0.79 \%$. The Swiss pulmonary hypertension registry consulted in December 2016 did not report additional CTEPH cases in these patients. The survey yielded $100 \%$ sensitivity and $81.6 \%$ specificity. The second step echocardiography in newly dyspnoeic patients showed a negative predictive value of $100 \%$.

CTEPH is a rare but treatable disease. A simple and sensitive way for CTEPH screening in patients with acute pulmonary embolism is recommended.

This article has supplementary material available from erj.ersjournals.com

Received: Dec 042017 | Accepted after revision: Feb 192018

Copyright OERS 2018 


\section{Introduction}

Chronic thromboembolic pulmonary hypertension (CTEPH) is viewed as a long-term complication of acute pulmonary embolism. Although its physiopathology remains poorly understood, the hypothesis relies on fibrotic transformation of thrombi in pulmonary arteries leading to nonhomogeneous vascular obstructions. Together with an overflow arteriopathy in the nonobstructed vascular bed, this causes an increase of the pulmonary artery pressure and, finally, right heart failure [1].

The cardinal symptom is progressive dyspnoea on exertion [2]. When oral anticoagulation was the only available treatment option, the prognosis was poor [3]. Pulmonary endarterectomy is nowadays a well-established therapy that has the potential to improve haemodynamics and survival [4]. Moreover, for the patient ineligible for surgery or with recurrent pulmonary hypertension after surgery there are new therapeutic options available: balloon pulmonary angioplasty and medical therapy or both together are increasingly used with benefits to the haemodynamics and quality of life [5-7]. Therefore, CTEPH can be considered as an uncommon, but serious and potentially curable complication of the frequently occurring pulmonary embolism [8].

Incidence of CTEPH after acute pulmonary embolism is currently a matter of debate and epidemiological data from large prospective cohorts of patients with acute pulmonary embolism are lacking. As background for our study, in 2008, published reports on the cumulative incidence of CTEPH after pulmonary embolism varied almost five-fold, from $0.8 \%$ to $3.8 \%$ [9-11]. Recently, a meta-analysis from ENDE-VERHAAR et al. [12] summed up current knowledge of this topic. They stratified previous studies according to their inclusion/exclusion criteria. Lower incidence is observed in unselected populations ("all comers") compared to pulmonary embolism survivors or pulmonary embolism survivors without major comorbidity. Therefore, a precise description of the studied population is essential for data analysis and comparison. In Switzerland, incidence of CTEPH can be only estimated from the Swiss Pulmonary Hypertension Registry (SPHR), a registry developed in 1998 to capture and follow-up patients with pulmonary hypertension [13].

The diagnosis of CTEPH is challenging, as symptoms are nonspecific. According to the current literature, CTEPH is often diagnosed with a delay of several months after the first symptom $[2,14,15]$. A systematic screening algorithm of patients following a pulmonary embolism event could be helpful for an earlier diagnosis of CTEPH and to identify cases with milder symptoms [16]. However, there is currently a lack of evidence in favour of any routine screening after pulmonary embolism $[4,17]$.

The study aims were to prospectively assess CTEPH incidence in patients diagnosed with pulmonary embolism and to test the usefulness of a multistep screening algorithm based on an initial dyspnoea questionnaire. In addition, we aimed to identify potential risk factors for developing CTEPH.

\section{Methods}

\section{Study participants}

This prospective, multicentre study was performed between March 2009 and November 2016 in 11 pulmonary hypertension centres in Switzerland. Patients were screened for acute pulmonary embolism and included in the study if the pulmonary embolism was confirmed by either pulmonary angiography, contrast-enhanced computed tomography (CT) or ventilation/perfusion $\left(V^{\prime} / Q^{\prime}\right)$ scan within the 4 weeks preceding the enrolment visit. All included patients signed informed consent. Patients were excluded if they were diagnosed before screening with pulmonary hypertension, pre-existing severe chronic dyspnoea New York Heart Association functional class (NYHA FC) III-IV, cancer or other threatening diseases with a life expectancy of $<6$ months. In addition, we excluded patients in whom it was not possible to assess NYHA FC due to severe mobility limitation. Irrespective of their final enrolment in the study, all patients screened were registered (initials, sex and date of birth).

\section{Outcomes}

The primary end-point was the incidence rate of CTEPH after acute pulmonary embolism. The secondary end-points were the assessment of the usefulness of a multistep screening algorithm and the identification of risk factors associated with development of CTEPH. To test the usefulness of the algorithm, we conducted a post hoc analysis matching the initial 1699 patients with pulmonary embolism with the data of the SPHR. The match was performed in December 2016 using patient initials, sex and date of birth. We first checked that patients within the study and diagnosed with CTEPH were listed in the SPHR. We then queried the SPHR for incident cases of CTEPH registered during the study period and looked-for individuals among the 1699 screened patients.

\section{Procedures}

A baseline health survey was completed at the enrolment visit. This questionnaire focused on demographics, baseline status and potential risk factors for pulmonary embolism or CTEPH. Pulmonary 


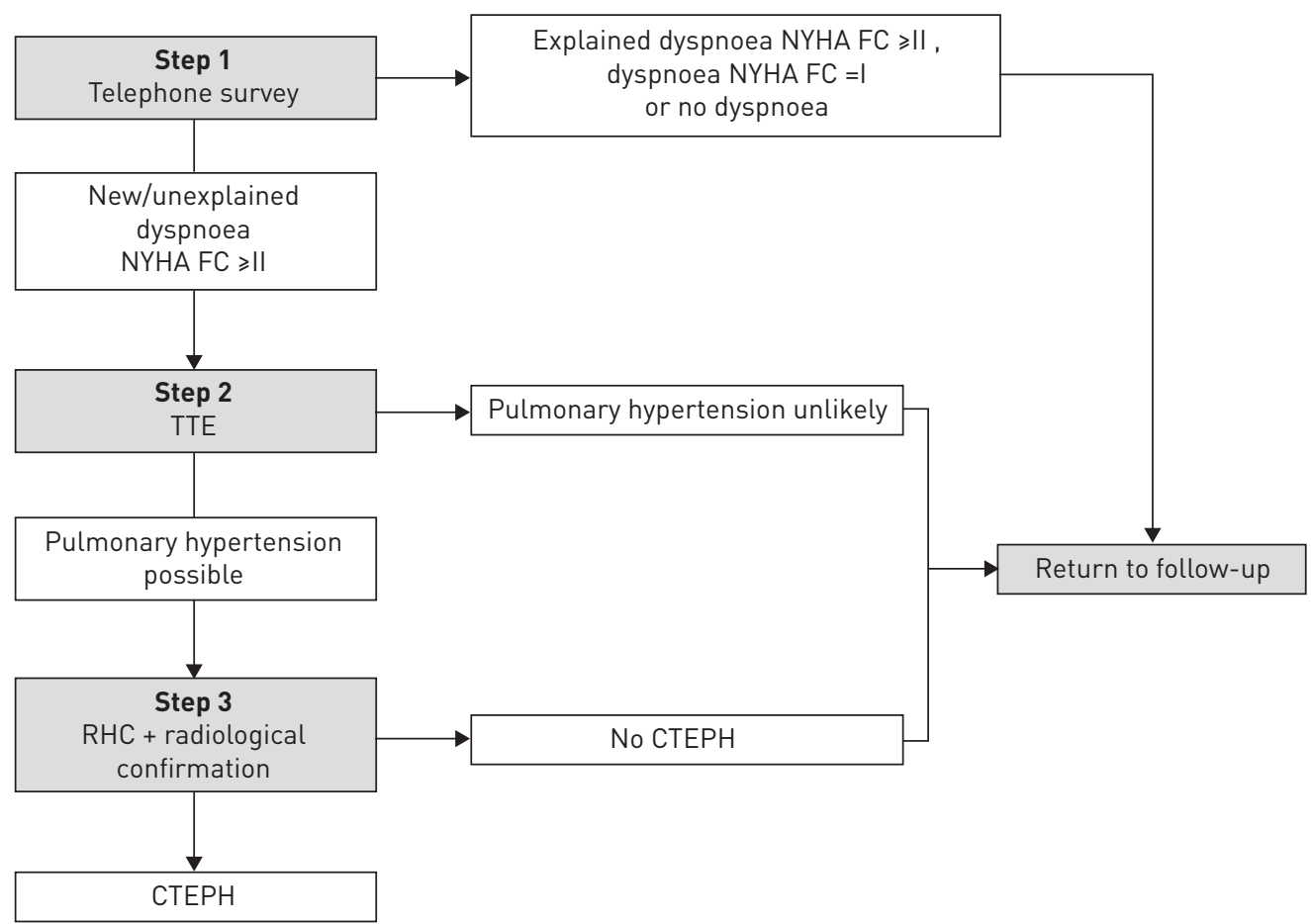

FIGURE 1 Algorithm of follow-up repeated at 6, 12 and 24 months. NYHA FC: New York Heart Association functional class; TTE: transthoracic echocardiography; RHC: right heart catheterisation; CTEPH: chronic thromboembolic pulmonary hypertension.

embolism therapy, including the choice and duration of anticoagulation was left to the discretion of physicians in charge according to local practice. We used the terms "provoked" and "unprovoked" pulmonary embolism, defined by the presence or absence, respectively, of one of the previously defined pulmonary embolism risk factors [18].

A three-step algorithm was created and applied at 6,12 and 24 months (figure 1). Step one was a phone assessment of dyspnoea, based on a standardised NYHA FC questionnaire translated into German, French and Italian (online supplementary material). If the dyspnoea score equalled NYHA FC II or greater, the patient advanced to the second step, unless an obvious and/or transient known cause that explained the current dyspnoea was identified. Step two consisted of a hospital visit for clinical examination, unblinded reassessment of the NYHA FC and transthoracic echocardiography (TTE). Based on TTE results, patients were classified as "pulmonary hypertension unlikely" or "pulmonary hypertension possible". These two groups were adapted over time from the 2004 and 2009 European guidelines [19, 20]. Pulmonary hypertension was considered possible if the peak tricuspid regurgitation velocity (TRV) was $>2.8 \mathrm{~m} \cdot \mathrm{s}^{-1}$ or if TRV was not measurable or $\leqslant 2.8 \mathrm{~m} \cdot \mathrm{s}^{-1}$ but other signs of pulmonary hypertension were present at TTE. If TRV was $\leqslant 2.8 \mathrm{~m} \cdot \mathrm{s}^{-1}$ and there were no other signs of pulmonary hypertension, pulmonary hypertension was considered unlikely and the patient returned to follow-up. In addition, TTE ordered by patient's general practitioner outside the study was accepted if above variables were assessable. If pulmonary hypertension was deemed possible, patients was engaged to step three for assessment by right heart catheterisation (RHC). According to the accepted definition of CTEPH, our diagnosis criteria were mean pulmonary arterial pressure $(\mathrm{mPAP}) \geqslant 25 \mathrm{mmHg}$, postcapillary wedge pressure $<15 \mathrm{mmHg}$, $\geqslant 3$ months of effective anticoagulation therapy and radiological confirmation with either $V^{\prime} / Q^{\prime}$ scan, contrast-enhanced CT or pulmonary angiography.

\section{Statistical analysis}

A sample size of 1000 patients was estimated in order to obtain a $2 \%$ wide $95 \%$ confidence interval for an expected CTEPH incidence after pulmonary embolism of 3\%. Incidence rate of CTEPH after pulmonary embolism was expressed as number of events in number of patient-years and cumulative incidence rate in percentage over 2 years. Descriptive statistics are presented as mean \pm SD for continuous data and as $\mathrm{n}(\%)$ and Wilson 95\% CI for categorical data. We calculated the percentage of concordance of the NYHA FC stage between the phone-based survey and the clinical evaluation. Accuracy of the screening algorithm was assessed by comparing it to the data of the SPHR using sensitivity, specificity and negative and positive 
predictive value at each step of the algorithm. For the risk factors analysis, we used a two-tailed t-test and a Fisher exact test for continuous and categorical values, respectively. A significance limit was set at a p-value $<0.05$ and all tests were two-tailed.

Primary and secondary end-points were analysed in patients with complete data. Patients were considered to be lost to follow-up if they withdrew their consent or didn't complete the last follow-up appointment, at least. For the primary end-point, sensitivity analyses were performed to account for missing data using multiple imputation techniques, described elsewhere [21]. We used R 3.3.3 (www.r-project.org) with the package mice 2.30 and SPSS 24 (IBM, Armonk, NY, USA) for statistical analyses [22].

Swiss ethics committees approved this study in 2008; all patients included signed an informed consent form.

\section{Results}

\section{Patients}

Patients were included between March 2009 and November 2013, and the study was closed in November 2016. Overall, 1699 consecutive patients were diagnosed with acute pulmonary embolism and assessed for eligibility. Of those, 542 patients were excluded and 555 could not sign the informed consent (figure 2). For the remaining 602 patients, 94 did not complete the study because they were lost to follow-up ( $\mathrm{n}=51$ ), withdrew their consent $(n=7)$ or died during the study period $(n=36)$. The causes of deaths were neoplasia $(n=15 ; 42 \%)$, cardiovascular diseases $(n=5 ; 14 \%)$, infection $(n=3 ; 8 \%)$, suicide $(n=1 ; 3 \%)$, unknown reasons $(n=9 ; 25 \%)$ and the last three $(8 \%)$ were sudden deaths during the primary hospitalisation that could only be imputed to the acute pulmonary embolism. Thus, 508 patients had a full follow-up over a median 2 years. The baseline characteristics of these patients are described in table 1.

\section{Incidence of CTEPH}

Over 2 years of follow-up, four CTEPH cases were diagnosed in the cohort of 508 fully followed pulmonary embolism patients. A description of the haemodynamics of each CTEPH patient is provided in table 2 (additional clinical parameters are provided in the online supplementary material). The cumulative incidence of CTEPH was $0.79 \%$ (95\% CI $0.31-2.07 \%)$ over a median 2 years, which yields an incidence rate of 3.7 per 1000 patient-years (95\% CI 1.43-9.36 per 1000 patient-years). Among patients presenting with a dyspnoea NYHA FC $\geqslant$ II in the survey $(n=97)$, the cumulative incidence of CTEPH rose to $4.12 \%$

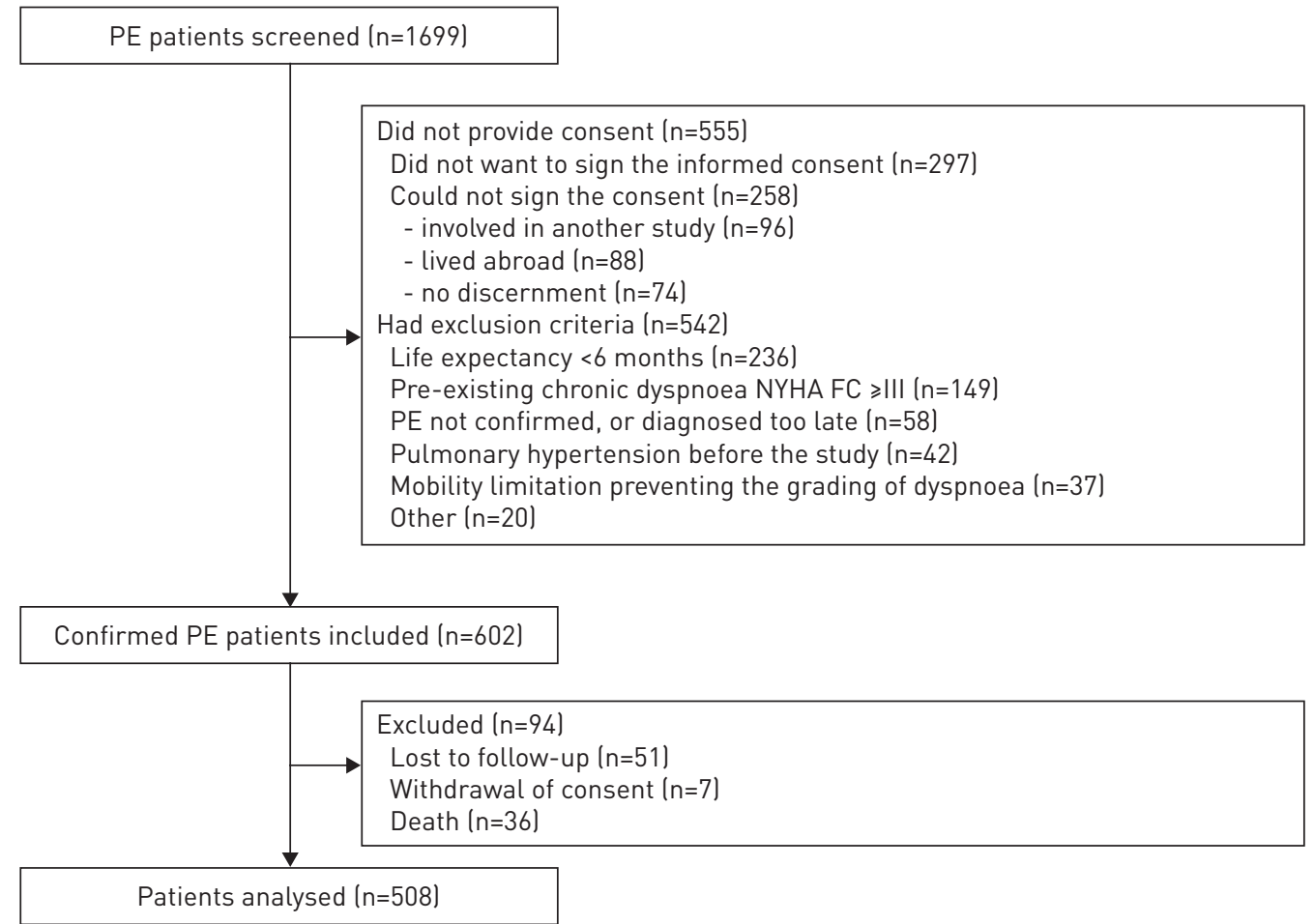

FIGURE 2 Patient selection profile. PE: pulmonary embolism; NYHA FC: New York Heart Association functional class. 


\section{TABLE 1 Baseline characteristics of the INPUT cohort}

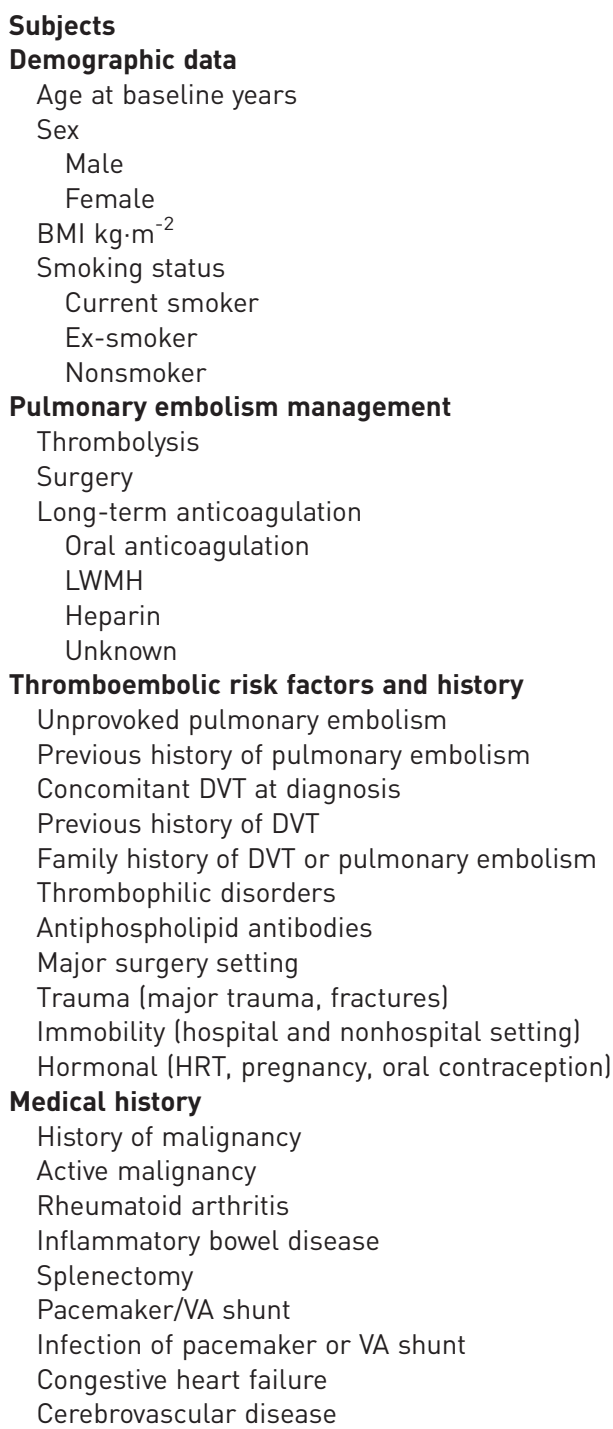

Data are presented as $n$, mean \pm SD or $n(\%)$. BMI: body mass index; LWMH: low-weight molecular heparin; DVT: deep vein thrombosis; HRT: hormone replacement therapy; VA: ventriculoatrial.

TABLE 2 Haemodynamics of the chronic thromboembolic pulmonary hypertension cases

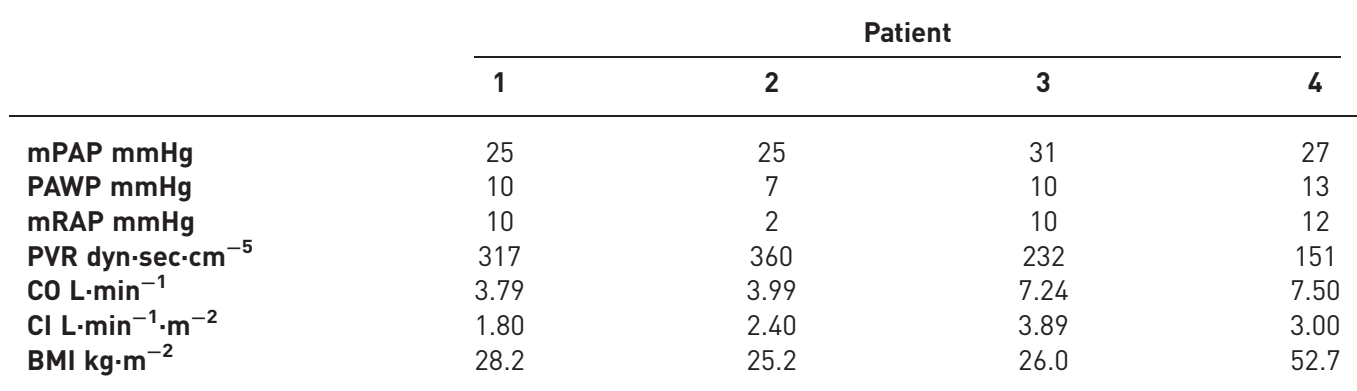

Data are presented as n. mPAP: mean pulmonary arterial pressure; PAWP: pulmonary arterial wedge pressure; mRAP: mean right atrial pressure; PVR: pulmonary vascular resistance; $\mathrm{CO}$ : cardiac output; $\mathrm{Cl}$ : cardiac index; BMI: body mass index. 
(95\% CI 1.62-10.13). Matching the 1699 screened patients with the SPHR identified four additional CTEPH cases among the 1097 excluded patients (causes of exclusion: involvement in other studies $(n=2)$, no discernment $(n=1)$, estimated life expectancy $<6$ months $(n=1))$. No other cases of CTEPH matching the identity of the 508 included patients under study were found in the SPHR. The sensitivity analyses led to similar incidence ranges.

\section{Screening algorithm}

The screening algorithm profile is described in figure 3. The phone-based dyspnoea survey identified 149 episodes of dyspnoea $\geqslant$ II NYHA FC in 97 (19.1\%) patients over the 2-year follow-up. The agreement of the NYHA FC between phone-based survey and clinical evaluation was $86.1 \%$ (95\% CI 78.1-91.6\%). The clinically evaluated NYHA FC class was higher than the phone-based survey in $8.2 \%$ (95\% CI 4.1-14.8\%) of these patients and lower in $6.2 \%$ (95\% CI 2.89-12.4\%).

TTE identified 15 episodes of possible pulmonary hypertension with a mean TRV of $2.96 \pm 0.05 \mathrm{~m} \cdot \mathrm{s}^{-1}$ in 14 patients who were invited for step 3. The RHC confirmed pulmonary hypertension in four patients with a mean mPAP of $27 \mathrm{mmHg}$. All four cases were CTEPH, confirmed either using $V^{\prime} / Q^{\prime}$ scanning $(n=3)$ or contrast-enhanced CT $(n=1)$. In four patients, RHC was not performed because of the patients' refusal $(n=3)$ or due to temporary contraindication $(n=1)$. However, for three of them, pulmonary hypertension was excluded using TTE at the next follow-up visit. The last patient refused to undergo RHC

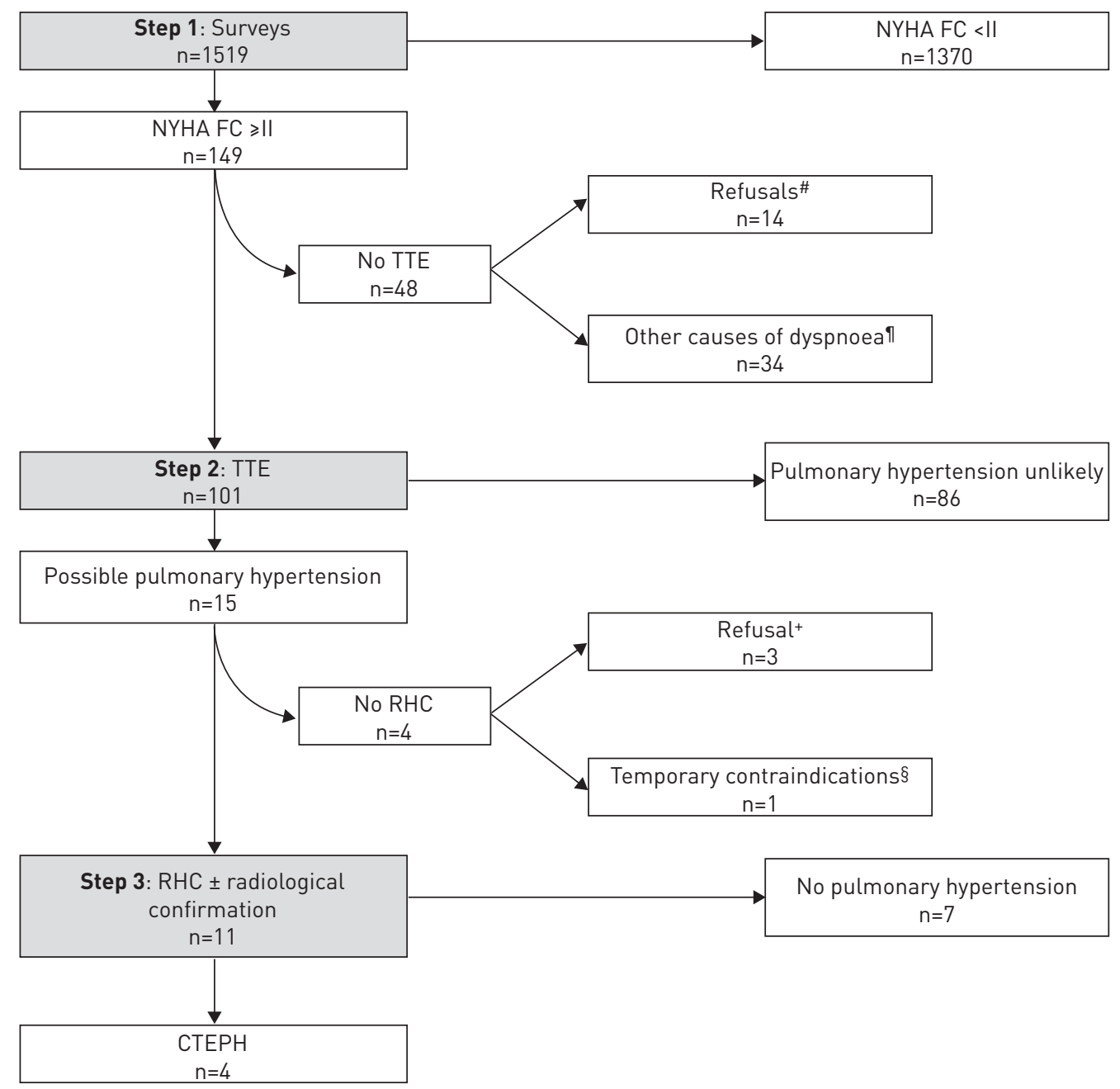

FIGURE 3 Screening algorithm profile. NYHA FC: New York Heart Association functional class; TTE: transthoracic echocardiography; RHC: right heart catheterisation; $\mathrm{CTEPH}$ : chronic thromboembolic pulmonary hypertension. \#: normal next follow-up appointment $(n=8)$ or last follow-up appointment $(n=6)$; " : respiratory causes $(n=17)$, obesity-related dyspnoeas $(n=5)$, ear, nose and throat causes $(n=5)$, lung cancer $(n=4)$, deconditioning-related dyspnoeas $(n=2)$, cardiac arrhythmia $(n=1) ;{ }^{+}$: pulmonary hypertension excluded using TTE at the next follow-up $(n=3) ;{ }^{\S}$ : patient clinically followed-up without sign of CTEPH $(n=1)$. 
TABLE 3 Accuracy of the screening algorithm for the survey in detecting chronic thromboembolic pulmonary hypertension at 6,12 and 24 months and over the 2-year period in all included pulmonary embolism patients and for transthoracic echocardiography (TTE) in the patients detected with dyspnoea

\begin{tabular}{lccccc} 
& $\mathbf{6}$ months & 12 months & 24 months & Overall survey & Overall TTE \\
\hline Subjects $\mathbf{n}$ & 508 & 506 & 505 & 508 & 97 \\
Sensitivity & 50 & 50 & 100 & 100 & 100 \\
Specificity & 88.9 & 91.5 & 90.9 & 81.6 & 88.7 \\
Positive predictive value & 3.4 & 2.3 & 2.1 & 4.1 & 26.7 \\
Negative predictive value & 99.6 & 99.8 & 100 & 100 & 100
\end{tabular}

Data are presented as \%, unless otherwise stated.

at the final follow-up visit, but was then regularly followed without signs of evolution towards a CTEPH over 6 years.

Accuracy of the screening algorithm compared to SPHR is described in table 3. The survey yielded $100 \%$ (95\% CI 51-100\%) sensitivity and $81.6 \%$ (95\% CI $77.9-88.4 \%)$ specificity. The second-step echocardiography in newly dyspnoeic patients showed a negative predictive value of $100 \%$ (95\% CI 51 $100 \%)$.

\section{Risk factors}

The presence of antiphospholipid antibodies was significantly associated with development of CTEPH after pulmonary embolism $(\mathrm{p}=0.03)$. No other risk factors were identified in all the other baseline characteristics tested (online supplementary material). A multivariate analysis was not applicable, due to small number of CTEPH cases.

\section{Discussion}

In this prospective observational study, we followed a large population of patients after acute pulmonary embolism. Our main finding is a cumulative incidence of CTEPH of $0.79 \%$ over 2 years. One in five patients will experience dyspnoea within 2 years following an acute pulmonary embolism. In these patients, the incidence of CTEPH rises to $4.12 \%$. Furthermore, our results show that our algorithm based on an initial dyspnoea assessment using NYHA FC is a sensitive way to screen pulmonary embolism patients for CTEPH. In addition, they confirm that the presence of antiphospholipid antibodies is a risk factor for the development of CTEPH after pulmonary embolism.

Compared to the existing literature, our study is the second largest multicentre cohort that has evaluated the incidence of CTEPH prospectively in patients with acute pulmonary embolism [12]. We found an incidence in the lower range of the previously published analogous studies $(0.4-9.1 \%)$ [9-11, 23-26]. The reason for such a wide range between studies may lie in the methodologies applied. The recent meta-analysis of ENDE-VERHAAR et al. [12] showed the impact of the selection criteria when considering the incidence of CTEPH after pulmonary embolism, distinguishing the three subgroups: "all comers", "survivors" and "survivors without pulmonary embolism". Our study may be classified as "survivors without major comorbidities", as we have performed a complete cases analysis and excluded some patients with severe comorbidities. Thus, we have a lower incidence than described in the meta-analysis for this subgroup $(2.8 \%, 95 \%$ CI $1.5-4.1 \%)$. The published studies included in this subgroup may have overestimated the incidence by the selective inclusion of higher-risk pulmonary embolism (notably unprovoked pulmonary embolism), while some could have misclassified acute pulmonary embolism as CTEPH $[27,28]$. We have addressed this latter issue with a post hoc control of CTEPH patient images to ensure that we did not miss any pre-existing pattern suggestive of CTEPH. Therefore, the risk of overestimation has been minimised. Conversely, we may face a possible underestimation through the negative segregation of high-risk patients, including the 236 with an estimated life expectancy $<6$ months and the 149 with a NYHA FC $\geqslant$ III. However, there were no significant differences in the sex and age distribution between the excluded and the included patients. Furthermore, the post hoc comparison to the SPHR showed a similar incidence range in included and excluded patients. This incidence is in the range of the "all comers" subgroup from the meta-analysis by ENDE-VERHAAR et al. (0.57\%, 95\% CI 0.13-0.98\%). This suggests an unbiased selection of patients. In addition, the higher incidence in published studies could be the consequence of the selection of patients only from tertiary high-volume centres that are probably prone to treating higher-risk pulmonary embolism. As we included patients from smaller 
hospitals and ambulatory patients, it is likely that we included more low-risk pulmonary embolism. Consequently, our results are more appropriate for generalisation to the entire population than previous reports.

We think that the present study is a valid assessment of the incidence of CTEPH after pulmonary embolism. First, we used recommended criteria to diagnose CTEPH, using strict RHC thresholds for pulmonary hypertension and standard radiological examinations [4]. As undertaken previously elsewhere, a senior specialist radiologist assessed the images of CTEPH patients and excluded a pre-existing pattern suggestive of CTEPH at the time of pulmonary embolism [27, 28]. It is now well established that all the studies that used TTE as the only diagnostic tool overestimated the incidence of CTEPH [12]. Furthermore, the match with the SPHR strengthens our findings. This registry gathers data from all recognised pulmonary hypertension centres in Switzerland and systematically collects all newly diagnosed CTEPH and pulmonary arterial hypertension cases [13]. Therefore, it allows us to confirm that we did not segregate a different subpopulation between the excluded and the included patients. In addition, it offers a good tool to eventually detect potential undiagnosed cases within the 2-year follow-up. We matched the 1699 screened patients from $\geqslant 3$ years and $<7$ years after the initial pulmonary embolism event. Given the natural history of the disease, all CTEPH cases, even with a honeymoon period of several months and a diagnosis delay of 2 years, should be symptomatic, diagnosed and listed in the registry [10, 26, 29]. In the SPHR, there was an average of 20.1 new cases of CTEPH per year between 2000 and 2012 [13]. With an estimated pulmonary embolism incidence rate of 0.6 per 1000 patient-years for a population of 8 million inhabitants, there are $\sim 5000$ acute pulmonary embolism events per year in Switzerland $[8,30]$. If we apply our CTEPH incidence rate to this number of pulmonary embolism events, we would expect 17.9 (95\% CI 7.2-46.8) new cases per year, which is close to the registry data. However, this calculation did not account for CTEPH cases without clinical pulmonary embolism, which could yield a slight lower number.

According to current literature, the diagnosis of CTEPH is often delayed [2, 15]. Most cases are diagnosed when patients reach NYHA FC III or IV [2]. If patients were diagnosed at an earlier stage, such as NYHA FC II, many would benefit from effective therapies [31]. Therefore, a screening strategy may be appealing for earlier diagnosis and treatment [31,32]. Presently, there is no official recommendation for any systematic screening in patients after pulmonary embolism. The only statement in the latest 2015 (European Society of Cardiology/European Respiratory Society) guidelines is to consider TTE in all patients with dyspnoea on exertion and history of pulmonary embolism [4]. In that sense, our algorithm represents a step further in favour of an active screening for CTEPH after pulmonary embolism.

With the algorithm applied in our study, we tested the sensitivity of a systematic screening based on a phone-based dyspnoea assessment within 2 years after acute pulmonary embolism. HeLD et al. [16] already showed in a smaller population that telephone symptom-based screening is valuable to identify CTEPH cases after pulmonary embolism. Furthermore, our algorithm is easily applicable in the real world as it is simple and conceivable for a general practitioner to follow patients with a practical dyspnoea survey over three visits within 2 years after acute pulmonary embolism. The first screening step, based on a symptomatic approach, is attractive because $>99 \%$ of CTEPH patients will develop dyspnoea [2]. Other algorithms based on risk factors could miss patients that nevertheless develop CTEPH in the absence of such risk factors. "CTEPH rule-out criteria" developed and externally validated by KLOK et al. [33] addresses the problem by including electrocardiographic features and N-terminal pro-brain natriuretic peptide value. Whether this strategy could be applied outside an experimental setting remains to be determined. Almost every published screening strategy uses TTE as a second step, since it is a noninvasive and widely accessible method to evaluate the presence of pulmonary hypertension. According to the low incidence of CTEPH, even in patients with dyspnoea, an efficient screening should yield the lowest false positive rate while false negatives should be near zero. Because of the high negative predictive value, present results support the use of TTE to select patients for RHC. Cardiopulmonary exercise testing is currently assessed in the diagnostic work-up, but until now its diagnostic performance is unknown and such a test could be difficult to apply widely [34]. Altogether, such a systematic screening may improve the awareness of CTEPH in patients with pulmonary embolism and favour earlier diagnosis.

This study has limitations. First, we did not reach the expected sample size. We decided to end enrolment because the number of positive cases was low and we had already achieved a precision aim of $2 \%$ wide 95\% CI for the primary end-point, which ensures the internal validity of the study. Second, we cannot exclude the possibility that some CTEPH cases remained undiagnosed. However, it would have been unethical and unrealistic to perform RHC in the 602 patients enrolled in the study. Nevertheless, the 2-year follow-up, together with the back-up control from the SPHR data appears to be a reasonable way to identify most symptomatic CTEPH cases. As 94 patients were lost to follow-up for various reasons, it is feasible that CTEPH cases went undetected in this population. To address this problem, we performed several sensitivity analyses which yielded similar results. Among 36 deaths, none had history of chronic 
right heart failure, although we acknowledge that post mortem examinations were not performed. We did not address the situation where a dyspnoeic patient is found with a normal resting haemodynamic at rest but with exercise pulmonary hypertension characterised by a steeper pressure-flow slope, as exercise RHC was not performed [35]. According to the current definition, such cases do not have CTEPH and are classified as chronic thromboembolic disease [36]. The prognosis and the indication to treat such cases remains a matter of debate. Third, we acknowledge a high proportion of excluded patients in the initially screened cohort. However, and unlike previous studies, the fate of the excluded patients has been documented though the SPHR, giving a reasonable estimate of symptomatic CTEPH cases in this population. Finally, we had designed the study a few months before the publication of the 2009 European guidelines [19]. Therefore, we had to slightly adapt the TTE criteria, which were initially based on the 2004 guidelines. Post hoc monitoring ensured that all 101 TTEs had been evaluated according to the latest guidelines with no change in patients that should have been invited for step 3.

In conclusion, CTEPH is a rare but devastating complication of pulmonary embolism. Our proposed algorithm is a simple and sensitive way to assess the development of CTEPH in such patients. We recommend that such systematic CTEPH screening should be performed regularly in the 2 years following a pulmonary embolism event for patients with new dyspnoea. Further research, including external validation and a cost-effectiveness analysis are needed to make this screening algorithm fully suitable for everyday clinical practice.

Acknowledgments: The authors sincerely thank Katharina Bruppacher (Actelion Pharma Schweiz, Baden, Switzerland) for her scientific input in developing the study protocol. In addition, we thank Claudia Tüller (Pneumologie, Lungenzentrum Hirslanden, Zurich, Switzerland) and Arnaud Perrier (Service de Médecine Interne Générale, Hôpitaux Universitaire Genevois, Geneva, Switzerland), former members of the steering committee, all the study nurses for their help in data collection and Sala Dine Qanadli (Service de Radiodiagnostic et Radiologie Interventionnelle, Centre Hospitalier Universitaire Vaudois, Lausanne, Switzerland) for reviewing all the radiology images of the chronic thromboembolic pulmonary hypertension patients. The authors are grateful to Denys Wahl (Actelion Pharma Schweiz) for editorial support.

This study is registered at www.isrctn.com with identifier number 61417303.

The study was funded by the Swiss Society for Pulmonary Hypertension. Funding information for this article has been deposited with the Crossref Funder Registry.

Conflict of interest: N. Coquoz received travel support for the international CTEPH conference 2017 (Leuven) from MSD Switzerland, and grants from Swiss society for pulmonary hypertension, during the conduct of the study. S. Ulrich has received grants from the Swiss National Science Foundation, Zurich Lung and Orpha Swiss, grants and personal fees from Actelion SA, Switzerland, and personal fees from MSD SA, Switzerland, outside the submitted work. J-D. Aubert received grants from the Swiss Society for Pulmonary Hypertension, during the conduct of the study; and personal fees from Bayer and Actelion Switzerland for advisory board participation, and travel expenses from Actelion Switzerland, outside the submitted work.

\section{References}

$1 \quad$ Piazza G, Goldhaber SZ. Chronic thromboembolic pulmonary hypertension. N Engl J Med 2011; 364: 351-360.

2 Pepke-Zaba J, Delcroix M, Lang I, et al. Chronic thromboembolic pulmonary hypertension (CTEPH): result from an international prospective study. Circulation 2011; 124: 1973-1981.

3 Lewczuk J, Piszko P, Jagas J, et al. Prognostic factors in medically treated patients with chronic pulmonary embolism. Chest 2001; 119: 818-823.

4 Galiè N, Humbert M, Vachiery J-L, et al. 2015 ESC/ERS Guidelines for the diagnosis and treatment of pulmonary hypertension. Eur Heart J 2016; 37: 67-119.

5 Lang I, Meyer BC, Ogo T, et al. Balloon pulmonary angioplasty in chronic thromboembolic pulmonary hypertension. Eur Respir Rev 2017; 26: 160119.

6 Pepke-Zaba J, Ghofrani H-A, Hoeper MM. Medical management of chronic thromboembolic pulmonary hypertension. Eur Respir Rev 2017; 26: 160107.

7 Madani M, Ogo T, Simonneau G. The changing landscape of chronic thromboembolic pulmonary hypertension management. Eur Respir Rev 2017; 26: 170105.

8 Oger E. Incidence of venous thromboembolism: a community-based study in western France. Thromb Haemost 2000; 83: 657-660

9 Becattini C, Agnelli G, Pesavento R, et al. Incidence of chronic thromboembolic pulmonary hypertension after a first episode of pulmonary embolism. Chest 2006; 130: 172-175.

10 Pengo V, Lensing AWA, Prins MH, et al. Incidence of chronic thromboembolic pulmonary hypertension after pulmonary embolism. N Engl J Med 2004; 350: 2257-2264.

11 Delcroix M, Kerr K, Fedullo P. Chronic thromboembolic pulmonary hypertension. Epidemiology and risk factors. Ann Am Thorac Soc 2016; 13: S201-S206.

12 Ende-Verhaar YM, Cannegieter SC, Noordegraaf AV, et al. Incidence of chronic thromboembolic pulmonary hypertension after acute pulmonary embolism: a contemporary view of the published literature. Eur Respir J 2017; 49: 1601792

13 Mueller-Mottet S, Stricker H, Domeninghetti G, et al. Long-term data from the Swiss pulmonary hypertension registry. Respiration 2015; 89: 127-140. 
14 Gall H, Hoeper MM, Richter MJ, et al. An epidemiological analysis of the burden of chronic thromboembolic pulmonary hypertension in the USA, Europe and Japan. Eur Respir Rev 2017; 26: 160121.

15 Held M, Grün M, Holl R, et al. Chronisch thromboembolische pulmonale Hypertonie: Latenz bis zur Diagnosesicherung und klinischer Zustand bei Diagnosestellung. [Chronic thromboembolic pulmonary hypertension: time delay from onset of symptoms to diagnosis and clinical condition at diagnosis]. Dtsch Med Wochenschr 2014; 139: 1647-1652.

16 Held M, Hesse A, Gött F, et al. A symptom-related monitoring program following pulmonary embolism for the early detection of CTEPH: a prospective observational registry study. BMC Pulm Med 2014; 14: 141.

17 Surie S, Gibson NS, Gerdes VEA, et al. Active search for chronic thromboembolic pulmonary hypertension does not appear indicated after acute pulmonary embolism. Thromb Res 2010; 125: e202-e205.

18 Bonderman D, Jakowitsch J, Adlbrecht C, et al. Medical conditions increasing the risk of chronic thromboembolic pulmonary hypertension. Thromb Haemost 2005; 93: 512-516.

19 Galiè N, Hoeper MM, Humbert M, et al. ESC/ERS Guidelines for the diagnosis and treatment of pulmonary hypertension. Eur Respir J 2009; 34: 1219-1263.

20 Galiè N, Torbicki A, Barst R, et al. Guidelines on diagnosis and treatment of pulmonary arterial hypertension. Eur Heart J 2004; 25: 2243-2278.

21 Rubin DB. Multiple Imputation for Nonresponse in Surveys. Hoboken, John Wiley \& Sons, 1987.

22 van Buuren S, Groothuis-Oudshoorn K. mice: Multivariate imputation by chained equations in R. J Stat Softw 2011; 45: 1-67.

23 Yang S, Yang Y, Zhai Z, et al. Incidence and risk factors of chronic thromboembolic pulmonary hypertension in patients after acute pulmonary embolism. J Thorac Dis 2015; 7: 1927-1938.

24 Klok FA, van Kralingen KW, van Dijk APJ, et al. Prospective cardiopulmonary screening program to detect chronic thromboembolic pulmonary hypertension in patients after acute pulmonary embolism. Haematologica 2010; 95: 970-975.

25 Martí D, Gómez V, Escobar C, et al. Incidencia de hipertensión pulmonar tromboembólica crónica sintomática y asintomática. [Incidence of symptomatic and asymptomatic chronic thromboembolic pulmonary hypertension]. Arch Bronconeumol 2010; 46: 628-633.

26 Klok FA, Zondag W, van Kralingen KW, et al. Patient outcomes after acute pulmonary embolism. A pooled survival analysis of different adverse events. Am J Respir Crit Care Med 2010; 181: 501-506.

27 Simonneau G, Hoeper MM. Evaluation of the incidence of rare diseases: difficulties and uncertainties, the example of chronic thromboembolic pulmonary hypertension. Eur Respir J 2017; 49: 1602522.

28 Guérin L, Couturaud F, Parent F, et al. Prevalence of chronic thromboembolic pulmonary hypertension after acute pulmonary embolism. Prevalence of CTEPH after pulmonary embolism. Thromb Haemost 2014; 112: 598-605.

29 Poli D, Grifoni E, Antonucci E, et al. Incidence of recurrent venous thromboembolism and of chronic thromboembolic pulmonary hypertension in patients after a first episode of pulmonary embolism. $J$ Thromb Thrombolysis 2010; 30: 294-299.

30 Kucera J, Krummenacher A. La Population de la Suisse 2015. [Switzerland's Population 2015]. Bern, Office Fédéral de la Statistique, 2016. www.bfs.admin.ch/bfs/fr/home/actualites/quoi-de-neuf.assetdetail.1401563.html

31 Ende-Verhaar YM, Huisman MV, Klok FA. To screen or not to screen for chronic thromboembolic pulmonary hypertension after acute pulmonary embolism. Thromb Res 2017; 151: 1-7.

32 Winter M-P, Schernthaner GH, Lang IM. Chronic complications of venous thromboembolism. J Thromb Haemost 2017; 15: 1531-1540.

33 Klok FA, Tesche C, Rappold L, et al. External validation of a simple non-invasive algorithm to rule out chronic thromboembolic pulmonary hypertension after acute pulmonary embolism. Thromb Res 2015; 135: 796-801.

34 Held M, Grün M, Holl R, et al. Cardiopulmonary exercise testing to detect chronic thromboembolic pulmonary hypertension in patients with normal echocardiography. Respiration 2014; 87: 379-387.

35 Herve P, Lau EM, Sitbon O, et al. Criteria for diagnosis of exercise pulmonary hypertension. Eur Respir J 2015; 46: 728-737.

36 Held M, Kolb P, Grün M, et al. Functional characterization of patients with chronic thromboembolic disease. Respiration 2016; 91: 503-509. 